\title{
Taller internacional: hacia la consolidación de Revistas de Geografía en América Latina
}

\author{
Carolina Negrete Rodríguez ${ }^{1}$
}

Con el objetivo de fomentar los lazos entre los equipos de trabajo de revistas de geografía de América Latina y los investigadores asociados a estos temas, Revista de Geografía Norte Grande ha decidido organizar un Taller Latinoamericano, en el que se invita a participar a directores y editores de revistas latinoamericanas especializadas en esta materia y a representantes de las redes internacionales relacionadas con revistas de corriente principal.

Este Taller Latinoamericano se organiza en el marco del Proyecto Realización de taller de revistas latinoamericanas de geografía y difusión de artículos preprint, recientemente aprobado por la Comisión Nacional de Investigación Científica y Tecnológica (CONICYT) de Chile. Dicho proyecto es parte del esfuerzo que ha realizado la Revista de Geografía Norte Grande durante los últimos años por lograr una cada vez mayor difusión del conocimiento científico que ella publica, junto a mejorar y mantener los estándares de calidad alcanzados a través del tiempo, que la han llevado a posicionarse como una de las más importantes revistas de geografía (de corriente principal) a nivel latinoamericano. Asimismo, forma parte del constante esfuerzo de fortalecimiento de las redes científicas latinoamericanas en temas geográficos y de la difusión de la experiencia adquirida en el proceso de indización e ingreso a importantes bases de datos.

La organización de este Taller Internacional estará a cargo del Instituto de Geografía de la Pontificia Universidad Católica de Chile, y contará con el auspicio de CONICYTChile y con el patrocinio de la Scientific Electronic Library Online, de Scielo-Chile y de la

\footnotetext{
1 Magíster (C) en Geografía y Geomática. Instituto de Geografía, Pontificia Universidad Católica de Chile (Chile).E-mail: cnegrete@geo.puc.cl
}

Red de Revistas Científicas de América Latina y el Caribe, España y Portugal (Redalyc).

Durante la actividad se ha propuesto cumplir con los siguientes objetivos específicos: recapitular los principales problemas que afectan a los editores científicos y analizar la forma de encararlos para lograr un mayor impacto, especialmente, respecto del ingreso a las indizaciones más importantes; intensificar las relaciones existentes entre las revistas de geografía y los investigadores sobre temas geográficos en los países involucrados; contribuir al mejoramiento de los procesos editoriales científicos de las revistas de geografía en América Latina y ampliar y fortalecer la difusión del conocimiento científico publicado en la colección histórica completa de la Revista de Geografía Norte Grande.

Además, se buscará avanzar en la discusión y difusión de algunos temas específicos, tales como: situación actual, alcance y dificultades que plantea el establecimiento de parámetros para indización en bases de datos y para el cálculo del factor de impacto; problemas relacionados con el diseño de bases de datos relativos a redes de cocitación de revistas y de autores; criterios para el mejoramiento de los niveles de calidad de las diversas revistas participantes; problemas y desafíos planteados por la transición desde el formato impreso al predominantemente virtual de libre acceso; problemas relacionados con la formalización y regularización de los derechos de autor; y situación relativa a la disponibilidad de árbitros para realizar peer review y sus efectos sobre la calidad de las publicaciones.

El encuentro se realizará los días 10 y 11 de noviembre de 2011, en la sede del Instituto de Geografía (Campus San Joaquín), de la Pontificia Universidad Católica de Chile. Ante consultas y confirmaciones, escribir al e-mail cnegrete@geo.puc.cl, o llamar al teléfono (56-2) - 3544750. 